\title{
Dual Orlicz mixed geominimal surface area
}

\author{
Li Gao ${ }^{a}$, Tongyi Mab,*, Yuanyuan $\mathrm{Guo}^{\mathrm{a}}$ \\ ${ }^{a}$ College of Mathematics and Statistics, Northwest Normal University, Lanzhou, Gansu, 730070, P. R. China. \\ ${ }^{b}$ College of Mathematics and Statistics, Hexi University, Zhangye, Gansu, 734000, P. R. China.
}

Communicated by J. Brzdek

\begin{abstract}
Based on the classical ideas, Zhu discussed the properties and useful theories for $\mathrm{L}_{\mathrm{p}}$-mixed geominimal surface area; meanwhile, Ma defined dual Orlicz geominimal surface area. The previous studies provide a thought to us for the study of the dual Orlicz mixed geominimal surface. In our paper, we have done the following work: attempting to use an integral form to define the dual Orlicz mixed geominimal surface area, further studyding its related properties, and listing some inequalities including Alexandrov-Fenchel type inequality, analogous cyclic inequality, Blaschke-Santaló type inequality, and affine isoperimetric inequality in Orlicz space.
\end{abstract}

Keywords: Star bodies, dual Orlicz mixed geominimal surface area, inequality.

2010 MSC: 52A20, 52A40.

(C)2018 All rights reserved.

\section{Introduction}

As early as the beginning of the 1970s, Petty defined early concept of the geominimal surface area $\mathrm{G}(\mathrm{K})$ (see [24]):

$$
\omega_{n}^{\frac{1}{n}} G(K)=\inf \left\{n V_{1}(K, Q) V\left(Q^{*}\right)^{\frac{1}{n}}: Q \in \mathcal{K}^{n}\right\}
$$

This definition is limited to $K \in \mathcal{K}^{n}$, and $\mathcal{K}^{n}$ is the set of convex bodies in $\mathbb{R}^{n}$. It's shown that $V_{1}(K, Q)$ stands for the mixed volume (see [12]). For more relevant detailed information, we can refer to the last two pages of this article (see $[8,29,30])$.

In the 1990's, the research of convex body theory has became more and more prosperous. Over the last two decades, a large number of research achievements in this field have sprung up and had great effects on other research fields. Lutwak, Yang and Zhang (See, for example, [10, 11, 13-15]) originated

\footnotetext{
*Corresponding author

Email address: matongyi@126.com (Tongyi Ma)
}

doi: $10.22436 /$ jnsa.011.10.01

Received: 2016-09-27 Revised: 2017-12-13 Accepted: 2018-04-14 
the $\mathrm{L}_{\mathrm{p}}$-Brunn-Minkowski-Firey theory. Subsequently, through the efforts of many scholars, the $\mathrm{L}_{\mathrm{p}}$-BrunnMinkowski theory (see [11]) has been put forward and some classical inequalities were proved, such as affine isoperimetric inequality (see [13]). Here, we provide some reference data for relevant applications (see, e.g., [23, 28, 32, 34, 36-38]).

Based on previous theoretical results and modeled on the definition of Petty, Lutwak proposed a mature concept, $G_{p}(K)$, as follows (see [11]): For $K \in K_{o}^{n}$,

$$
\omega_{n}^{\frac{p}{n}} G_{p}(K)=\inf \left\{n V_{p}(K, Q) V\left(Q^{*}\right)^{\frac{p}{n}}: Q \in \mathcal{K}_{o}^{n}\right\},
$$

here $\mathcal{K}_{\mathrm{o}}^{n}$ is such a convex body as contains the origin in its interior and $V_{p}(K, Q)$ stands for $L_{p}$-mixed volume (see [11]).

Orlicz space is a more wide function space than the $L_{p}$ space which we are familiar with. Since the Orlicz space was proposed and named in 1932, the theoretical system of the Orlicz space is gradually perfect under the unremitting efforts of many researchers. Meanwhile, many new research directions have been developed, which make the research of Orlicz space more abundant (see $[1,4,6,16-18,31,35]$ ). While calculation of areas in Orlicz space is one of the hot research issues, the results of the study are still weak. Finding effective ways is a key link in dealing with the issue of area calculation in Orlicz space. In Orlicz space, we usually let $\Phi$ define the set of convex functions $\phi:[0, \infty) \rightarrow[0, \infty)$ that satisfy $\phi(0)=0$, $\phi(1)=1$, and $\lim _{t \rightarrow \infty} \phi(t)=+\infty$. From this, the original concept of the Orlicz geominimal surface area shows that if $K \in \mathcal{K}_{\mathrm{o}}^{\mathfrak{n}}$ and $\phi \in \Phi$, then (see [33])

$$
\mathrm{G}_{\phi}(\mathrm{K})=\inf \left\{\mathrm{nV}_{\phi}(\mathrm{K}, \mathrm{Q}): \mathrm{Q} \in \mathcal{K}_{\mathrm{o}}^{\mathrm{n}} \text { and } \mathrm{V}\left(\mathrm{Q}^{*}\right)=\omega_{\mathrm{n}}\right\},
$$

where $\mathrm{V}_{\phi}(\mathrm{K}, \mathrm{Q})$ represents the Orlicz mixed volume (see [20]).

In 2016, Ma and Wang defined the dual Orlicz geominimal surface area as follows (see [20]): For $K \in \mathcal{S}_{\mathrm{o}}^{n}$,

$$
\tilde{\mathrm{G}}_{-\phi}(\mathrm{K})=\inf \left\{n \tilde{V}_{-\phi}(K, Q): Q \in \mathcal{K}_{o}^{n} \text { and } V\left(Q^{*}\right)=\omega_{n}\right\},
$$

here $\tilde{V}_{-\phi}(K, Q)$ represents the dual Orlicz mixed volume (see Section 2).

In addition, they came up with an important theory as follows: For $K \in \mathcal{S}_{\mathrm{o}}^{n}$ and $\phi \in \Phi$, Ma and Wang found a unique body $\tilde{\mathrm{K}} \in \mathcal{K}_{\mathrm{o}}^{\mathrm{n}}$ so that they get (see [20])

$$
\tilde{\mathrm{G}}_{-\phi}(\mathrm{K})=\mathrm{n} \tilde{\mathrm{V}}_{-\phi}(\mathrm{K}, \tilde{\mathrm{K}}) \text { and } \mathrm{V}\left(\tilde{\mathrm{K}}^{*}\right)=\omega_{\mathrm{n}} \text {. }
$$

Afterwards they found $\tilde{\mathrm{K}}=\tilde{\mathrm{T}}_{\phi} \mathrm{K}$, here $\tilde{\mathrm{K}}$ is the dual Orlicz-Petty body. Thus, for $\mathrm{K} \in \mathcal{S}_{\mathrm{o}}^{n}$, and $\phi \in \Phi$, we get (see [20])

$$
\tilde{\mathrm{G}}_{-\phi}(\mathrm{K})=n \tilde{\mathrm{V}}_{-\phi}\left(\mathrm{K}, \tilde{\mathrm{T}}_{\phi} \mathrm{K}\right) \text { and } \mathrm{V}\left(\tilde{\mathrm{T}}_{\phi}^{*} \mathrm{~K}\right)=\omega_{n} .
$$

From (1.2) and (2.2), Ma and Wang get the following integral representation of $\tilde{G}_{-\phi}(\mathrm{K})$. Obviously, for $K \in \mathcal{S}_{\mathrm{o}}^{n}$ and $\phi \in \Phi$, we get (see [20])

$$
\tilde{\mathrm{G}}_{-\phi}(\mathrm{K})=\int_{\mathrm{S}^{\mathrm{n}-1}} \phi\left(\frac{\rho_{\mathrm{K}}(\mathrm{u})}{\rho_{\tilde{\mathrm{T}}_{\phi} \mathrm{K}}}\right) \rho_{\mathrm{K}}^{\mathrm{n}}(\mathrm{u}) \mathrm{d} S(\mathrm{u}) .
$$

If $\mathrm{K} \in \mathcal{K}_{\mathrm{c}}^{\mathrm{n}}$, then, for $\phi \in \Phi$, (see [20])

$$
\tilde{\mathrm{G}}_{-\phi}(\mathrm{K}) \tilde{\mathrm{G}}_{-\phi}\left(\mathrm{K}^{*}\right) \leqslant\left(n \omega_{n}\right)^{2},
$$

with equality if and only if $\mathrm{K}$ is an ellipsoid.

We are going to finish this article in three parts. First, Section 1 is some related background information we introduced in the preface. Then, we list some definitions and results in Section 2, which will be applied in proofs in Section 3. Finally, we give our main results and their proofs in Section 3. 


\section{Notation and preliminaries}

In $\mathbb{R}^{n}, \mathcal{K}_{c}^{n}$ indicates that a kind of set full of convex bodies whose centroids lie at the origin. For any body $K, V(K)$ is its volume. Thus, $\omega_{n}=V(B)$ is equivalent to the volume for unit ball. Additionally, star bodies about the origins in $\mathbb{R}^{n}$ are denoted as $\mathcal{S}_{0}^{n}$.

For $K \in \mathbb{R}^{n}$, the radial function $\rho_{K}(u): \mathbb{R}^{\mathfrak{n}} \backslash 0 \rightarrow[0, \infty)$ is defined by

$$
\rho_{\mathrm{K}}(u)=\max \{\lambda \geqslant 0: \lambda u \in K\}, \quad u \in S^{n-1} .
$$

We call $K$ star body if $\rho_{K}$ is positive and continuous. Furthermore, if $\rho_{K}(u) / \rho_{L}(u)$ is independent of $u \in S^{n-1}$, we say the two star bodies $K$ and $L$ are dilates.

We also have some useful properties for $\rho_{K}$ as follows: For $\lambda>0$,

$$
\rho_{K}(\lambda x)=\lambda^{-1} \rho_{K}(x) \text { and } \rho_{\lambda K}(x)=\lambda \rho_{K}(x) .
$$

If a set $K \in \mathcal{K}_{0}^{n}$, then $K^{*}$, the polar body of $K$ is defined by (see $\left.[3,27]\right)$

$$
\mathrm{K}^{*}=\left\{x \in \mathbb{R}^{\mathrm{n}}: x \cdot \mathrm{y} \leqslant 1, \forall \mathrm{y} \in \mathrm{K}\right\} .
$$

For $K \in \mathcal{K}_{c}^{n}$, the Blaschke-Santaló inequality states that (see [21, 22])

$$
\mathrm{V}(\mathrm{K}) \mathrm{V}\left(\mathrm{K}^{*}\right) \leqslant \omega_{n}^{2},
$$

with equality if and only if $\mathrm{K}$ is an ellipsoid.

Integral formula for the dual Orlicz mixed volume is mentioned in [20] as follows:

$$
\tilde{V}_{-\phi}(\mathrm{K}, \mathrm{L})=\frac{1}{\mathrm{n}} \int_{\mathrm{S}^{n-1}} \phi\left(\frac{\rho_{\mathrm{K}}(\mathrm{u})}{\rho_{\mathrm{L}}(\mathrm{u})}\right) \rho_{\mathrm{K}}^{\mathrm{n}}(\mathrm{u}) \mathrm{d} S(\mathrm{u}) .
$$

Apparently, we have

$$
\tilde{\mathrm{V}}_{-\phi}(\mathrm{K}, \mathrm{K})=\mathrm{V}(\mathrm{K})
$$

They also established the following dual Orlicz-Minkowski inequality: Suppose $\phi \in \Phi$. If $K, L \in \mathcal{S}_{o}^{n}$, then (see [20])

$$
\tilde{\mathrm{V}}_{-\phi}(\mathrm{K}, \mathrm{L}) \geqslant \mathrm{V}(\mathrm{K}) \phi\left(\left(\frac{\mathrm{V}(\mathrm{K})}{\mathrm{V}(\mathrm{L})}\right)^{\frac{1}{n}}\right) .
$$

Here, we try to get the generalized isoperimetric inequality for the dual Orlicz geominimal surface area.

Lemma 2.1. If $\phi \in \Phi$, for $\mathrm{K} \in \mathcal{K}_{\mathrm{c}}^{\mathrm{n}}$, then

$$
\tilde{G}_{-\phi}(K) \leqslant \frac{n \omega_{n}^{2}}{V\left(K^{*}\right)} \phi\left(\left(\frac{\omega_{n}}{V\left(K^{*}\right)}\right)^{\frac{1}{n}}\right),
$$

with equality only if $\mathrm{K}$ is an ellipsoid.

Proof. If $\mathrm{Q}=\lambda \mathrm{K}$, then $\mathrm{V}\left(\mathrm{Q}^{*}\right)=\omega_{\mathrm{n}}$. Thus $\lambda=\left(\frac{\mathrm{V}\left(\mathrm{K}^{*}\right)}{\left(\omega_{\mathrm{n}}\right)}\right)^{\frac{1}{n}}$. Motivated by definitions of $\tilde{\mathrm{G}}_{-\phi}(\mathrm{K})$ and $\tilde{V}_{-\phi}(K, Q)$, meanwhile the Blaschke-Santaló inequality (2.1), we identify that

$$
\begin{aligned}
\tilde{\mathrm{G}}_{-\phi}(\mathrm{K}) \leqslant n \tilde{V}_{-\phi}(\mathrm{K}, \lambda \mathrm{K}) & =\int_{S^{n-1}} \phi\left(\frac{\rho_{\mathrm{K}}(\mathrm{u})}{\rho_{\lambda \mathrm{K}}(\mathrm{u})}\right) \rho_{\mathrm{K}}^{\mathrm{n}}(\mathrm{u}) \mathrm{dS}(\mathrm{u}) \\
& =n \phi(1 / \lambda) \mathrm{V}(\mathrm{K})=\mathrm{nV}(\mathrm{K}) \phi\left(\left(\frac{\omega_{n}}{\mathrm{~V}\left(\mathrm{~K}^{*}\right)}\right)^{\frac{1}{n}}\right) \leqslant \frac{n \omega_{n}^{2}}{\mathrm{~V}\left(\mathrm{~K}^{*}\right)} \phi\left(\left(\frac{\omega_{n}}{\mathrm{~V}\left(\mathrm{~K}^{*}\right)}\right)^{\frac{1}{n}}\right) .
\end{aligned}
$$

Clearly the equality holds only when $\mathrm{K}$ is an ellipsoid. 
Compared with the inequality (2.3) and Ma's $L_{p}$-affine isoperimetric inequality, if $\phi(t)=|t|^{p}$, and $p \geqslant 1$, then they become the same as follows (see [19]):

$$
\tilde{G}_{-p}(K) \leqslant n \omega_{n}^{(2 n-p) / n} V\left(K^{*}\right)^{-(n+p) / n},
$$

with equality if and only if $\mathrm{K}$ is a ball centered at the origin.

Based on the above proof of the Lemma 2.1, and using the reverse Blaschke-Santalo inequality (see $[5,25])$,

$$
\mathrm{V}(\mathrm{K}) \mathrm{V}\left(\mathrm{K}^{*}\right) \geqslant \frac{4^{\mathrm{n}}}{\mathrm{n} !}
$$

with equality if and only if $\mathrm{K}$ is zonoids, we can get the following lemma.

Lemma 2.2. If $\phi \in \Phi$, for $\mathrm{K} \in \mathcal{K}_{\mathrm{c}}^{\mathrm{n}}$, then

$$
\tilde{\mathrm{G}}_{-\phi}(\mathrm{K}) \leqslant n V(K) \phi\left(\left(\frac{4^{n} \omega_{n}}{(n !) V(K)}\right)^{\frac{1}{n}}\right) .
$$

The following Hölder's integral inequality will be used (see [2, 7]).

Lemma 2.3. Let $\mathrm{f}_{0}, \mathrm{f}_{1}, \ldots, \mathrm{f}_{\mathrm{k}}$ be Borel measurable functions on $\mathrm{X}$. Suppose that $\mathrm{p}_{0}, \mathrm{p}_{1}, \ldots, \mathrm{p}_{\mathrm{k}}$ are nonzero numbers with $\sum_{i=1}^{k} \frac{1}{p_{i}}=1$. Then

$$
\int_{X} f_{0}(u) f_{1}(u) \cdots f_{k}(u) d u \leqslant \prod_{i=1}^{k}\left(\int_{X} f_{0}(u) f_{i}(u)^{p_{i}} d u\right)^{\frac{1}{p_{i}}},
$$

with equality if and only if either there are constants $\mathrm{b}_{1}, \mathrm{~b}_{2}, \ldots, \mathrm{b}_{\mathrm{k}}$ not all zero, such that

$$
b_{1}\left|f_{1}(u)\right|^{p_{1}}=b_{2}\left|f_{2}(u)\right|^{p_{2}}=\ldots b_{k}\left|f_{k}(u)\right|^{p_{k}},
$$

or one of the functions is null.

\section{Main results and proofs}

Ma and Feng introduced the general concept of dual $\mathrm{L}_{p}$-mixed geominimal surface area for star bodies, and established some affine isoperimetric inequalities in [19]. On this basis, we give a definition for the dual Orlicz mixed geominimal surface area in the same way. Here $\tilde{G}_{-\phi}\left(K_{1}, \ldots, K_{n}\right)$ used to express the new concept named the dual Orlicz mixed geominimal surface area. For $K_{1}, \ldots, K_{n} \in \mathcal{S}_{o}^{n}$, we have:

Definition 3.1. If $K_{1}, \ldots, K_{n} \in \mathcal{S}_{o}^{n}$, then there exists a unique body (dual Orlicz-Petty body of $\left.K_{i}\right) \tilde{T}_{\phi} K(i=$ $1, \ldots, n)$ with

$$
\tilde{\mathrm{G}}_{-\phi}\left(\mathrm{K}_{1}, \ldots, \mathrm{K}_{\mathrm{n}}\right)=\int_{S^{n-1}}\left[\phi\left(\frac{\rho_{\mathrm{K}_{1}}(\mathrm{u})}{\rho_{\tilde{\mathrm{T}}_{1}}(\mathrm{u})}\right) \rho_{\mathrm{K}_{1}}^{\mathrm{n}}(\mathrm{u}) \cdots \phi\left(\frac{\rho_{\mathrm{K}_{n}}(\mathrm{u})}{\rho_{\tilde{\mathrm{T}}_{n}}(\mathrm{u})}\right) \rho_{\mathrm{K}_{n}}^{n}(\mathrm{u})\right]^{\frac{1}{n}} \mathrm{~d} S(u) .
$$

Suppose $\tilde{g}_{-\phi}\left(K_{i}, u\right)=\phi\left(\frac{\rho_{K_{i}}(u)}{\rho_{\tilde{T}_{i}}(u)}\right) \rho_{K_{i}}^{n}(u)$, then the above formula can be expressed as follows:

$$
\tilde{\mathrm{G}}_{-\phi}\left(\mathrm{K}_{1}, \ldots, \mathrm{K}_{n}\right)=\int_{\mathrm{S}^{n-1}}\left[\tilde{\mathrm{g}}_{-\phi}\left(\mathrm{K}_{1}, \mathrm{u}\right) \cdots \tilde{\mathrm{g}}_{-\phi}\left(\mathrm{K}_{n}, \mathrm{u}\right)\right]^{\frac{1}{n}} \mathrm{dS}(\mathrm{u})
$$

The classical dual Aleksandrov-Fenchel inequality for dual mixed volume states that if $K_{1}, \ldots, K_{n} \in S_{o}^{n}$, then $($ see $[3,9,26])$

$$
\tilde{V}\left(K_{1}, \ldots, K_{n}^{m}\right) \leqslant \prod_{i=0}^{m-1} \tilde{V}(K_{1}, \ldots, K_{n-m}, \underbrace{K_{n-i}, \ldots, K_{n-i}}_{m}),
$$

with equality if and only if $K_{n-m+1}, K_{n-m+2}, \ldots, K_{n}$ are dilates. 
Now, we prove the Alexandrov-Fenchel type inequality for dual Orlicz mixed geominimal surface area as follows.

Theorem 3.2. For $\mathrm{K}_{1}, \ldots, \mathrm{K}_{\mathrm{n}} \in \mathcal{S}_{\mathrm{o}}^{\mathrm{n}}$, suppose $1 \leqslant \mathrm{~m}<\mathrm{n}$, then

$$
\tilde{G}_{-\phi}\left(K_{1}, \ldots, K_{n}\right)^{m} \leqslant \prod_{i=0}^{m-1} \tilde{G}_{-\phi}(K_{1}, \ldots, K_{n-m}, \underbrace{K_{n-i}, \ldots, K_{n-i}}_{m}),
$$

with equality if the $\mathrm{K}_{\mathrm{j}}$ are dilates for $\mathrm{j}=\mathrm{n}-\mathrm{m}+1, \ldots, \mathrm{n}$. For $\mathrm{m}=1$, equality holds trivially.

In particular, for $\mathrm{m}=\mathrm{n}$, we have

$$
\tilde{\mathrm{G}}_{-\phi}\left(\mathrm{K}_{1}, \ldots, \mathrm{K}_{\mathrm{n}}\right)^{\mathrm{n}} \leqslant \tilde{\mathrm{G}}_{-\phi}\left(\mathrm{K}_{1}\right) \cdots \tilde{\mathrm{G}}_{-\phi}\left(\mathrm{K}_{\mathrm{n}}\right),
$$

equality holds if the $\mathrm{K}_{i}$ are dilates.

Proof. Let $Y_{0}(u)=\left[\tilde{g}_{-\phi}\left(K_{1}, u\right) \cdots \tilde{g}_{-\phi}\left(K_{n-m}, u\right)\right]^{\frac{1}{n}}$ and $Y_{i+1}(u)=\left[\tilde{g}_{-\phi}\left(K_{n-i}, u\right)\right]^{\frac{1}{n}}$, for $i=0, \ldots, m-1$, from (3.1) and Lemma 2.3, we get

$$
\begin{aligned}
& \tilde{\mathrm{G}}_{-\phi}\left(\mathrm{K}_{1}, \ldots, \mathrm{K}_{n}\right)=\int_{\mathrm{S}^{n-1}}\left[\tilde{\mathrm{g}}_{-\phi}\left(\mathrm{K}_{1}, \mathfrak{u}\right) \cdots \tilde{\mathrm{g}}_{-\phi}\left(\mathrm{K}_{n}, \mathrm{u}\right)\right]^{\frac{1}{\mathrm{n}}} \mathrm{dS}(\mathrm{u}) \\
& =\int_{S^{n-1}} Y_{0}(u) Y_{1}(u) \cdots Y_{m}(u) d S(u) \\
& \leqslant \prod_{i=0}^{m-1}\left(\int_{S^{n-1}} Y_{0}(u) Y_{i+1}(u)^{m} d S(u)\right)^{\frac{1}{m}} \\
& =\prod_{i=0}^{m-1} \tilde{G}_{-\phi}^{\frac{1}{m}}(K_{1}, \ldots, K_{n-m}, \underbrace{K_{n-i}, \ldots, K_{n-i}}_{m}) \text {. }
\end{aligned}
$$

For every $c_{i j}>0$ and all $0 \leqslant i \neq j \leqslant m-1$, according to the condition of equality in Lemma 2.3, we get the equality of the Theorem 3.2 holds if and only if $Y_{0}(u) Y_{i+1}^{m}(u)=c_{i j}^{m} Y_{0}(u) Y_{j+1}^{m}(u)$. This is equivalent to

$$
\phi\left(\frac{\rho_{K_{n-i}}(u)}{\rho_{\tilde{T}_{\phi} K_{n-i}}(u)}\right) \rho_{K_{n-i}}^{n}(u)=c_{i j} \phi\left(\frac{\rho_{K_{n-j}}(u)}{\rho_{\tilde{T}_{\phi} K_{n-j}}(u)}\right) \rho_{K_{n-j}}^{n}(u) ;
$$

one can observe taht the equality holds if $K_{n-i}$ and $K_{n-j}$ are dilates.

Next, we will introduce the concept of $i$ th dual Orlicz mixed geominimal surface area and discuss related inequalities about $\tilde{G}_{-\phi, i}(K, L)$, which extend the dual Orlicz mixed geominimal surface area.

If $K, L \in \mathcal{S}_{0}^{n}$ with $i \in \mathbb{R}$, then we define

$$
\tilde{\mathrm{G}}_{-\phi, i}(\mathrm{~K}, \mathrm{~L})=\int_{S^{n-1}} \tilde{\mathrm{g}}_{-\phi}(\mathrm{K}, \mathrm{u})^{\frac{\mathrm{n}-\mathrm{i}}{n}} \tilde{g}_{-\phi}(\mathrm{L}, \mathrm{u})^{\frac{i}{\mathrm{i}}} \mathrm{dS}(\mathrm{u}) .
$$

From (1.2), we get

$$
\tilde{G}_{-\phi}(B)=n \tilde{V}_{-\phi}\left(B, \tilde{T}_{\phi} B\right)
$$

Since

$$
\tilde{\mathrm{G}}_{-\phi}(\mathrm{B})=n \omega_{\mathrm{n}}=n \tilde{\mathrm{V}}_{-\phi}(\mathrm{B}, \mathrm{B}),
$$

together (3.3), (3.4) with the uniqueness part of (1.1), we have

$$
\tilde{\mathrm{T}}_{\phi} \mathrm{B}=\mathrm{B} \text {. }
$$


Let $\mathrm{L}=\mathrm{B}$ and write

$$
\tilde{\mathrm{G}}_{-\phi, i}(\mathrm{~K}, \mathrm{~B})=\tilde{\mathrm{G}}_{-\phi, i}(\mathrm{~K}) \text {. }
$$

Combining (3.2) and (3.5), and noticing that $\rho_{\tilde{T}_{\phi} B}=\rho_{B}=1$, we have

$$
\tilde{\mathrm{G}}_{-\phi, i}(\mathrm{~K})=\int_{\mathrm{S}^{n-1}} \tilde{\mathrm{g}}_{-\phi}(\mathrm{K}, \mathrm{u})^{\frac{\mathrm{n}-\mathrm{i}}{\mathrm{i}}} \mathrm{d} \mathrm{S}(\mathrm{u}) .
$$

Consequently, by (3.1), (3.2), and (3.5), we get

$$
\begin{array}{ll}
\tilde{\mathrm{G}}_{-\phi, 0}(\mathrm{~K}, \mathrm{~B})=\tilde{\mathrm{G}}_{-\phi}(\mathrm{K}), \quad \tilde{\mathrm{G}}_{-\phi, i}(\mathrm{~K}, \mathrm{~K})=\tilde{\mathrm{G}}_{-\phi}(\mathrm{K}), \\
\tilde{\mathrm{G}}_{-\phi, 0}(\mathrm{~K}, \mathrm{~L})=\tilde{\mathrm{G}}_{-\phi}(\mathrm{K}), \quad \tilde{\mathrm{G}}_{-\phi, \mathrm{n}}(\mathrm{K}, \mathrm{L})=\tilde{\mathrm{G}}_{-\phi}(\mathrm{L}),
\end{array}
$$

The following Theorem deals with the cyclic inequality for the ith dual Orlicz mixed geominimal surface.

Theorem 3.3. For $K, L \in \mathcal{S}_{0}^{n}$, if $i, j, k \in \mathbb{R}$ and $i<j<k$, then

$$
\tilde{\mathrm{G}}_{-\phi, i}(K, L)^{k-j} \tilde{\mathrm{G}}_{-\phi, k}(K, L)^{j-i} \geqslant \tilde{\mathrm{G}}_{-\phi, j}(K, L)^{k-i},
$$

equality holds if $\mathrm{K}$ and $\mathrm{L}$ are dilates.

Proof. From Definition 3.2 and Hölder's integral inequality (see Lemma 2.3), we get

$$
\begin{aligned}
\tilde{G}_{-\phi, i}(K, L)^{\frac{k-j}{k-i}} \tilde{G}_{-\phi, k}(K, L)^{\frac{j-i}{k-i}}= & {\left[\int_{S^{n-1}} \tilde{g}_{-\phi}(K, u)^{\frac{n-i}{n}} \tilde{g}_{-\phi}(L, u)^{\frac{i}{n}} \mathrm{dS}(u)\right]^{\frac{k-j}{k-i}} } \\
& \times\left[\int_{S^{n-1}} \tilde{g}_{-\phi}(K, u)^{\frac{n-k}{n}} \tilde{g}_{-\phi}(L, u)^{\frac{k}{n}} \mathrm{~d} S(u)\right]^{\frac{j-i}{k-i}} \\
= & \left\{\int_{S^{n-1}}\left[\tilde{g}_{-\phi}(K, u)^{\frac{(n-i)(k-j)}{n(k-i)}} \tilde{g}_{-\phi}(L, u)^{\frac{i(k-j)}{n(k-i)}}\right]^{\frac{k-i}{k-j}} \mathrm{dS}(u)\right\}^{\frac{k-j}{k-i}} \\
& \times\left\{\int_{S^{n-1}}\left[\tilde{g}_{-\phi}(K, u)^{\frac{(n-k)(j-i)}{n(k-i)}} \tilde{g}_{-\phi}(L, u)^{\frac{k(j-i)}{n(k-i)}}\right]^{\frac{k-i}{j-i}} d S(u)\right\}^{\frac{j-i}{k-i}} \\
\geqslant & \int_{S^{n-1}} \tilde{g}_{-\phi}(K, u)^{\frac{n-j}{n}} \tilde{g}_{-\phi}(L, u)^{\frac{j}{n}} d S(u)=\tilde{G}_{-\phi, j}(K, L) .
\end{aligned}
$$

We proved inequality (3.8). Apparently, the equality in (3.8) holds true if and only if for any $u \in S^{n-1}$,

$$
\frac{\tilde{g}_{-\phi}(K, u)^{\frac{n-i}{n}} \tilde{g}_{-\phi}(L, u)^{\frac{i}{n}}}{\tilde{g}_{-\phi}(K, u)^{\frac{n-k}{n}} \tilde{g}_{-\phi}(L, u)^{\frac{k}{n}}}
$$

is a constant. It also implies that for any $u \in S^{n-1}, \tilde{g}_{-\phi}(K, u) / \tilde{g}_{-\phi}(L, u)$ is a constant. By the similar argument as in the proof of Theorem 3.2, we conclude that equality in (3.8) holds if $\mathrm{K}$ and L are dilates.

If $\mathrm{L}=\mathrm{B}$, and combining with (3.5), a direct consequence of Theorem 3.3 is obtained:

Corollary 3.4. For $K, L \in \mathcal{S}_{0}^{n}$, if $i, j, k \in \mathbb{R}$ and $i<j<k$, then

$$
\tilde{\mathrm{G}}_{-\phi, i}(\mathrm{~K})^{\mathrm{k}-\mathrm{j}} \tilde{\mathrm{G}}_{-\phi, k}(\mathrm{~K})^{j-i} \geqslant \tilde{\mathrm{G}}_{-\phi, j}(\mathrm{~K})^{\mathrm{k}-\mathrm{i}},
$$

equality holds if $\mathrm{K}$ is a ball with centroid at the origin. 
Then, we obtain the Minkowski inequality for the dual ith Orlicz mixed geominimal surface area as follows.

Theorem 3.5. If $K, L \in \mathcal{S}_{0}^{n}$, for $i \in \mathbb{R}$, then, for $i<0$ or $i>n$,

$$
\tilde{\mathrm{G}}_{-\phi, i}(\mathrm{~K}, \mathrm{~L})^{\mathrm{n}} \geqslant \tilde{\mathrm{G}}_{-\phi}(\mathrm{K})^{\mathrm{n}-\mathrm{i}} \tilde{\mathrm{G}}_{-\phi}(\mathrm{L})^{\mathrm{i}},
$$

and for $0<\mathrm{i}<\mathrm{n}$,

$$
\tilde{\mathrm{G}}_{-\phi, i}(\mathrm{~K}, \mathrm{~L})^{\mathrm{n}} \leqslant \tilde{\mathrm{G}}_{-\phi}(\mathrm{K})^{\mathrm{n}-\mathrm{i}} \tilde{\mathrm{G}}_{-\phi}(\mathrm{L})^{\mathrm{i}} .
$$

Equality of each inequality holds if $\mathrm{K}$ and $\mathrm{L}$ are dilates. For $\mathrm{i}=0$ or $\mathrm{i}=\mathrm{n}$, above inequalities are identical.

Proof.

(i). For $i<0$, suppose $(i, j, k)=(i, 0, n)$ in Theorem 3.3, we get

$$
\tilde{\mathrm{G}}_{-\phi, i}(\mathrm{~K}, \mathrm{~L})^{\mathrm{n}} \tilde{\mathrm{G}}_{-\phi, n}(\mathrm{~K}, \mathrm{~L})^{-i} \geqslant \tilde{\mathrm{G}}_{-\phi, 0}(\mathrm{~K}, \mathrm{~L})^{\mathrm{n}-\mathrm{i}},
$$

equality holds if $\mathrm{K}$ and $\mathrm{L}$ are dilates.

From (3.7), we have

$$
\tilde{\mathrm{G}}_{-\phi, i}(\mathrm{~K}, \mathrm{~L})^{\mathrm{n}} \tilde{\mathrm{G}}_{-\phi}(\mathrm{L})^{-i} \geqslant \tilde{\mathrm{G}}_{-\phi}(\mathrm{K})^{\mathrm{n}-\mathrm{i}},
$$

i.e.,

$$
\tilde{\mathrm{G}}_{-\phi, i}(\mathrm{~K}, \mathrm{~L})^{\mathrm{n}} \geqslant \tilde{\mathrm{G}}_{-\phi}(\mathrm{K})^{\mathrm{n}-\mathrm{i}} \tilde{\mathrm{G}}_{-\phi}(\mathrm{L})^{\mathrm{i}},
$$

equality holds if $\mathrm{K}$ and $\mathrm{L}$ are dilates.

(ii). For $i>n$, let $(i, j, k)=(0, n, i)$ in Theorem 3.3 , we get

$$
\tilde{\mathrm{G}}_{-\phi, 0}(\mathrm{~K}, \mathrm{~L})^{\mathrm{i}-\mathrm{n}} \tilde{\mathrm{G}}_{-\phi, i}(\mathrm{~K}, \mathrm{~L})^{\mathrm{n}} \geqslant \tilde{\mathrm{G}}_{-\phi, n}(\mathrm{~K}, \mathrm{~L})^{\mathrm{i}},
$$

with equality holds if $\mathrm{K}$ and $\mathrm{L}$ are dilates.

From (3.7), we have

$$
\tilde{\mathrm{G}}_{-\phi}(\mathrm{K})^{i-n} \tilde{\mathrm{G}}_{-\phi, i}(\mathrm{~K}, \mathrm{~L})^{\mathrm{n}} \geqslant \tilde{\mathrm{G}}_{-\phi}(\mathrm{L})^{\mathrm{i}},
$$

i.e.,

$$
\tilde{\mathrm{G}}_{-\phi, i}(\mathrm{~K}, \mathrm{~L})^{\mathrm{n}} \geqslant \tilde{\mathrm{G}}_{-\phi}(\mathrm{K})^{\mathrm{n}-\mathrm{i}} \tilde{\mathrm{G}}_{-\phi}(\mathrm{L})^{\mathrm{i}},
$$

equality holds if $\mathrm{K}$ and $\mathrm{L}$ are dilates.

(iii). For $0<i<n$, let $(i, j, k)=(0, i, n)$ in Theorem 3.3, we get

$$
\tilde{\mathrm{G}}_{-\phi, o}(K, L)^{\mathrm{n}-\mathrm{i}} \tilde{\mathrm{G}}_{-\phi, n}(\mathrm{~K}, \mathrm{~L})^{\mathrm{i}} \geqslant \tilde{\mathrm{G}}_{-\phi, i}(\mathrm{~K}, \mathrm{~L})^{\mathrm{n}},
$$

equality holds if $\mathrm{K}$ and $\mathrm{L}$ are dilates.

From (3.7), we have inequality (3.10).

(iv). For $i=0$ (or $i=n$ ), by (3.7), inequality (3.10) (or (3.9)) is identical.

Taking $L=B$ in Theorem 3.5, using (3.5) and $\tilde{G}_{-\phi}(B)=n \omega_{n}$, we immediately obtain the following corollary. 
Corollary 3.6. If $\mathrm{K} \in \mathcal{S}_{\mathrm{o}}^{n}$, for $i \in \mathbb{R}$, then, for $i<0$ or $i>n$,

$$
\tilde{\mathrm{G}}_{-\phi, i}(K)^{n} \geqslant\left(n \omega_{n}\right)^{i} \tilde{\mathrm{G}}_{-\phi}(K)^{n-i},
$$

and for $0<\mathrm{i}<\mathrm{n}$,

$$
\tilde{\mathrm{G}}_{-\phi, i}(K)^{n} \leqslant\left(n \omega_{n}\right)^{i} \tilde{\mathrm{G}}_{-\phi}(K)^{n-i} .
$$

Equality of each inequality holds true if $\mathrm{K}$ is a ball with centroid at the origin. For $i=0$ or $i=n$, above inequalities are identical.

Then, we prove the Blaschke-Santaló type inequality for dual Orlicz mixed geominimal surface area.

Theorem 3.7. If $\mathrm{K}, \mathrm{L} \in \mathcal{K}_{\mathrm{c}}^{\mathrm{n}}$, for $\mathrm{i} \in \mathbb{R}$ and $0 \leqslant i \leqslant n$, then

$$
\tilde{\mathrm{G}}_{-\phi, i}(\mathrm{~K}, \mathrm{~L}) \tilde{\mathrm{G}}_{-\phi, i}\left(K^{*}, \mathrm{~L}^{*}\right) \leqslant\left(n \omega_{n}\right)^{2} .
$$

For $0<i<n$, equality holds if $\mathrm{K}$ and $\mathrm{L}$ are ellipsoids with dilation of each other. For $\mathrm{i}=0$ (or $\mathrm{i}=\mathrm{n}$ ), the inequality holds as an equality if $\mathrm{K}$ is an ellipsoid centered at the origin.

Proof. For $0<i<n$, by (3.10) and (1.3), we get

$$
\tilde{\mathrm{G}}_{-\phi, i}(\mathrm{~K}, \mathrm{~L})^{\mathrm{n}} \tilde{\mathrm{G}}_{-\phi, i}\left(\mathrm{~K}^{*}, \mathrm{~L}^{*}\right)^{n} \leqslant\left[\tilde{\mathrm{G}}_{-\phi}(\mathrm{K}) \tilde{\mathrm{G}}_{-\phi}\left(\mathrm{K}^{*}\right)\right]^{\mathrm{n}-\mathrm{i}}\left[\tilde{\mathrm{G}}_{-\phi}(\mathrm{L}) \tilde{\mathrm{G}}_{-\phi}\left(\mathrm{L}^{*}\right)\right]^{i}=\left(n \omega_{n}\right)^{2 n} .
$$

That is,

$$
\tilde{\mathrm{G}}_{-\phi, i}(\mathrm{~K}, \mathrm{~L}) \tilde{\mathrm{G}}_{-\phi, i}\left(\mathrm{~K}^{*}, \mathrm{~L}^{*}\right) \leqslant\left(n \omega_{n}\right)^{2},
$$

equality holds true if $\mathrm{K}$ and $\mathrm{L}$ are ellipsoids with dilation of each other.

For $i=0$ (or $i=n$ ), it follows from (3.7), (3.10) (or (3.9)), and (1.3) that Theorem 3.7 is obvious, and with equality true if $\mathrm{K}$ (or L) is an ellipsoid.

Theorem 3.8. If $K, L \in \mathcal{K}_{c}^{n}$, for $i \in \mathbb{R}$, then

(i) for $0 \leqslant i \leqslant n$,

$$
\tilde{\mathrm{G}}_{-\phi, i}(\mathrm{~K}) \tilde{\mathrm{G}}_{-\phi, i}\left(K^{*}\right) \leqslant\left(n \omega_{n}\right)^{2},
$$

equality holds true if $\mathrm{K}$ is a ball;

(ii) for $i \geqslant n$,

$$
\tilde{G}_{-\phi, i}(K) \tilde{G}_{-\phi, i}\left(K^{*}\right) \geqslant\left(n \omega_{n}\right)^{2},
$$

equality holds true if $\mathrm{K}$ is a ball.

Proof.

(i). By Theorem 3.7, letting $L=B$, we get

$$
\tilde{\mathrm{G}}_{-\phi, i}(\mathrm{~K}, \mathrm{~B}) \tilde{\mathrm{G}}_{-\phi, i}\left(\mathrm{~K}^{*}, \mathrm{~B}^{*}\right) \leqslant\left(n \omega_{n}\right)^{2},
$$

by (3.5),

$$
\tilde{\mathrm{G}}_{-\phi, i}(\mathrm{~K}) \tilde{\mathrm{G}}_{-\phi, i}\left(\mathrm{~K}^{*}\right) \leqslant\left(n \omega_{n}\right)^{2},
$$

equality holds true if $\mathrm{K}$ is a ball. 
(ii). For all $i \geqslant n$, by inequality (3.11), we have

$$
\left(\frac{\tilde{\mathrm{G}}_{-\phi, i}(\mathrm{~K})}{\tilde{\mathrm{G}}_{-\phi, i}(\mathrm{~B})}\right)^{\mathrm{n}} \geqslant\left(\frac{\tilde{\mathrm{G}}_{-\phi}(\mathrm{K})}{\tilde{\mathrm{G}}_{-\phi}(\mathrm{B})}\right)^{\mathrm{n}-\mathrm{i}} .
$$

From (3.13), $\tilde{\mathrm{G}}_{-\phi}(\mathrm{B})=n \omega_{n}$, and (1.3), we obtain

$$
\left(\frac{\tilde{\mathrm{G}}_{-\phi, i}(\mathrm{~K}) \tilde{\mathrm{G}}_{-\phi, i}\left(\mathrm{~K}^{*}\right)}{\tilde{\mathrm{G}}_{-\phi, i}(\mathrm{~B})^{2}}\right)^{n} \geqslant\left(\frac{\tilde{\mathrm{G}}_{-\phi}(\mathrm{K}) \tilde{\mathrm{G}}_{-\phi}\left(\mathrm{K}^{*}\right)}{\tilde{\mathrm{G}}_{-\phi}(\mathrm{B})^{2}}\right)^{\mathrm{n}-\mathrm{i}} \geqslant 1 .
$$

That is

$$
\tilde{\mathrm{G}}_{-\phi, i}(\mathrm{~K}) \tilde{\mathrm{G}}_{-\phi, i}\left(\mathrm{~K}^{*}\right) \geqslant\left(n \omega_{n}\right)^{2},
$$

equality holds true if $\mathrm{K}$ is a ball.

Theorem 3.9. If $K, L \in \mathcal{K}_{c}^{n}$, for $i \in \mathbb{R}$, then

(i) for $0 \leqslant i \leqslant n$,

$$
\frac{\tilde{\mathrm{G}}_{-\phi, i}(\mathrm{~K})}{\tilde{\mathrm{G}}_{-\phi, i}(\mathrm{~B})} \leqslant\left(\frac{\mathrm{V}(\mathrm{B})}{\mathrm{V}\left(\mathrm{K}^{*}\right)} \phi\left(\left(\frac{\omega_{n}}{\mathrm{~V}\left(\mathrm{~K}^{*}\right)}\right)^{\frac{1}{n}}\right)\right)^{\frac{\mathrm{n}-\mathrm{i}}{n}},
$$

equality holds true if $\mathrm{K}$ is a ball;

(ii) for $i \geqslant n$,

$$
\frac{\tilde{\mathrm{G}}_{-\phi, i}(\mathrm{~K})}{\tilde{\mathrm{G}}_{-\phi, i}(\mathrm{~B})} \geqslant\left(\frac{\mathrm{V}(\mathrm{B})}{\mathrm{V}\left(\mathrm{K}^{*}\right)} \phi\left(\left(\frac{\omega_{n}}{\mathrm{~V}\left(\mathrm{~K}^{*}\right)}\right)^{\frac{1}{n}}\right)\right)^{\frac{n-i}{n}},
$$

equality holds true if $\mathrm{K}$ is a ball.

Proof.

(i). For $i=0$ with (3.6) and Lemma 2.1, we have

$$
\frac{\tilde{\mathrm{G}}_{-\phi}(\mathrm{K})}{\tilde{\mathrm{G}}_{-\phi}(\mathrm{B})} \leqslant \frac{\frac{n \omega_{n}^{2}}{\mathrm{~V}\left(\mathrm{~K}^{*}\right)} \phi\left(\left(\frac{\omega_{n}}{\mathrm{~V}\left(\mathrm{~K}^{*}\right)}\right)^{\frac{1}{n}}\right)}{\frac{n \omega_{n}^{2}}{\mathrm{~V}(\mathrm{~B})} \phi\left(\left(\frac{\omega_{n}}{V(B)}\right)^{\frac{1}{n}}\right)}=\frac{V(B)}{V\left(K^{*}\right)} \phi\left(\left(\frac{\omega_{n}}{V\left(K^{*}\right)}\right)^{\frac{1}{n}}\right),
$$

equality holds if $\mathrm{K}$ is a ball.

For $i=n$, by (3.5) and (3.7), the equality holds trivially.

For $0<i<n$, by (3.12) and Lemma 2.1, we get

$$
\left(\frac{\tilde{\mathrm{G}}_{-\phi, i}(\mathrm{~K})}{\tilde{\mathrm{G}}_{-\phi, i}(\mathrm{~B})}\right)^{n} \leqslant\left(\frac{\tilde{\mathrm{G}}_{-\phi}(\mathrm{K})}{\tilde{\mathrm{G}}_{-\phi}(\mathrm{B})}\right)^{n-i} \leqslant\left(\frac{\mathrm{V}(\mathrm{B})}{\mathrm{V}\left(\mathrm{K}^{*}\right)} \phi\left(\left(\frac{\omega_{n}}{V\left(\mathrm{~K}^{*}\right)}\right)^{\frac{1}{n}}\right)\right)^{n-i}
$$

equality holds true if $\mathrm{K}$ is a ball.

(ii). For $i>n$, by (3.11) and Lemma 2.1, we get

$$
\left(\frac{\tilde{G}_{-\phi, i}(K)}{\tilde{G}_{-\phi, i}(B)}\right)^{n} \geqslant\left(\frac{\tilde{G}_{-\phi}(K)}{\tilde{G}_{-\phi}(B)}\right)^{n-i} \geqslant\left(\frac{V(B)}{V\left(K^{*}\right)} \phi\left(\left(\frac{\omega_{n}}{V\left(K^{*}\right)}\right)^{\frac{1}{n}}\right)\right)^{n-i},
$$

equality holds true if $\mathrm{K}$ is a ball. This proof is completed. 
From Lemma 2.2 and Corollary 3.6, and using the same argument as in the proof of Theorem 3.9, we can also obtain the following results.

Theorem 3.10. If $K, \mathrm{~L} \in \mathcal{K}_{\mathrm{c}}^{\mathrm{n}}$, for $i \in \mathbb{R}$, then

(i) for $0 \leqslant i \leqslant n$,

$$
\frac{\tilde{\mathrm{G}}_{-\phi, i}(\mathrm{~K})}{\tilde{\mathrm{G}}_{-\phi, i}(\mathrm{~B})} \leqslant\left(\frac{\mathrm{V}(\mathrm{K})}{\mathrm{V}(\mathrm{B})} \phi\left(\left(\frac{4^{\mathrm{n}} \omega_{n}}{(\mathrm{n} !) \mathrm{V}(\mathrm{K})}\right)^{\frac{1}{n}}\right)\right)^{\frac{\mathrm{n}-\mathrm{i}}{\mathrm{n}}}
$$

(ii) for $i \geqslant n$,

$$
\frac{\tilde{\mathrm{G}}_{-\phi, i}(K)}{\tilde{\mathrm{G}}_{-\phi, i}(\mathrm{~B})} \geqslant\left(\frac{\mathrm{V}(\mathrm{K})}{\mathrm{V}(\mathrm{B})} \phi\left(\left(\frac{4^{\mathrm{n}} \omega_{n}}{(\mathrm{n} !) \mathrm{V}(\mathrm{K})}\right)^{\frac{1}{n}}\right)\right)^{\frac{n-i}{n}}
$$

\section{Acknowledgment}

This research is supported by the National Natural Science Foundation of China (Grant No. 11561020).

\section{References}

[1] F. W. Chen, J. Z. Zhou, C. Y. Yang, On the reverse Orlicz Busemann-Petty centroid inequality, Adv. in Appl. Math., 47 (2011), 820-828. 1

[2] H. Federer, Geometric Measure Theory, Springer-Verlag, New York, (1969). 2

[3] R. J. Gardner, Geometric Tomography (second edition), Cambridge University Press, New York, (2006). 2, 3

[4] R. J. Gardner, D. Hug, W. Weil, The Orlicz-Brunn-Minkowski theory: a general framework, additions, and inequalities, J. Differential Geom., 97 (2014), 427-476. 1

[5] Y. Gordon, M. Meyer, S. Reisner, Zonoids with minimal volume product: A new proof, Proc. Amer. Math. Soc., 104 (1988), 273-276. 2

[6] C. Haberl, E. Lutwak, D. Yang, G. Y. Zhang, The even Orlicz Minkowski problem, Adv. Math., 224 (2010), $2485-2510$. 1

[7] G. Hardy, J. Littlewood, G. Pólya, inequalities, Cambridge University Press, London, (1934). 2

[8] M. Ludwig, M. Reitzner, A characterization of affine surface area, Adv. Math., 147 (1999), 138-172. 1

[9] M. Ludwig, C. Schütt, E. Werner, Approximation of the Euclidean ball by polytopes, Studia Math., 173 (2006), 1-18. 3

[10] E. Lutwak, The Brunn-Minkowski-Firey theory. I. Mixed volumes and the Minkowski problem, J. Differential Geom., 38 (1993), 131-150. 1

[11] E. Lutwak, The Brunn-Minkowski-Firey theory II: affine and geominimal surface areas, Adv. Math., 118 (1996), $244-294$. 1

[12] E. Lutwak, Volume of mixed bodies, Trans. Am. Math. Soc., 294 (1986), 487-500. 1

[13] E. Lutwak, D. Yang, G. Y. Zhang, $\mathrm{L}_{\mathrm{p}}$ affine isoperimetric inequalities, J. Differential Geom., 56 (2000), 111-132. 1

[14] E. Lutwak, D. Yang, G. Y. Zhang, Sharp affine $\mathrm{L}_{p}$ Sobolev inequalities, J. Differential Geom., 62 (2002), 17-38.

[15] E. Lutwak, D. Yang, G. Y. Zhang, On the $\mathrm{L}_{\mathrm{p}}$-Minkowski problem, Trans. Amer. Math. Soc., 356 (2004), 4359-4370. 1

[16] E. Lutwak, D. Yang, G. Y. Zhang, Orlicz centroid bodies, J. Differential Geom., 84 (2010), 365-387. 1

[17] E. Lutwak, D. Yang, G. Y. Zhang, Orlicz projection bodies, Adv. Math., 223 (2010), $220-242$.

[18] T. Y. Ma, The Minimal Dual Orlicz Surface Area,Taiwanese J. Math., 20 (2016), 287-309. 1

[19] T. Y. Ma, Y. B. Feng, Dual L $\mathrm{L}_{\mathrm{p}}$-Mixed Geominimal Surface Area and Related Inequalities, J. Funct. Spaces, 2016 (2016), 10 pages. 2,3

[20] T. Y. Ma, W. D. Wang, Dual Orlicz geominimal surface area, J. Inequal. Appl., 2016 (2016), 13 pages. 1, 1, 1, 2, 2

[21] M. Meyer, A. Pajor, On Santalós inequality, in: Geometric Aspects of Functional Analysis, 1989 (1989), 261-263. 2

[22] M. Meyer, A. Pajor, On the Blaschke-Santalós inequality, Arch. Math., 55 (1990), 82-93. 2

[23] M. Moszyńska, Quotient Star Bodies, Intersection Bodies and Star Duality, J. Math. Anal. Appl., 232 (1999), 45-60. 1

[24] C. M. Petty, Geominimal surface area, Geometriae Dedicata, 3 (1974), 77-97. 1

[25] S. Reisner, Zonoids with minimal volume product, Math. Z., 192 (1986), 339-346. 2

[26] R. Schneider, Convex Bodies: The Brunn-Minkowski Theory (Second ed.), Cambridge University Press, Cambridge, (2014). 3

[27] R. Schneider, Convex Bodies: The Brunn-Minkowski Theory, Cambridge University Press, Cambridge, (1993). 2

[28] W. D. Wang, Y. B. Feng, A general Lp-version of Petty's affine projection inequality, Taiwanese J. Math., 17 (2013), 517-528. 1

[29] E. Werner, On L -affine surface areas, Indiana Univ. Math. J., 56 (2007), 2305-2323. 1 


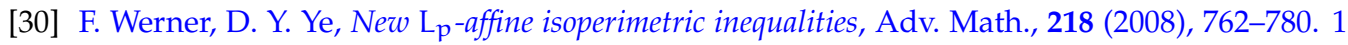

[31] D. M. Xi, H. L. Jin, G. S. Leng, The Orlicz Brunn-Minkowski inequality, Adv. Math., 260 (2014), 350-374. 1

[32] J. Yuan, W.-S. Cheung, Lp intersection bodies, J. Math. Anal. Appl., 338 (2008), 1431-1439. 1

[33] S. F. Yuan, H. L. Jin, G. S. Leng, Orlicz geominimal surface areas, Math. Inequal. Appl., 18 (2015), $353-362.1$

[34] C.-J. Zhao, G. S. Leng, Brunn-Minkowski inequality for mixed intersection bodies, J. Math. Anal. Appl., 301 (2005), 115-123. 1

[35] G. X. Zhu, The Orlicz centroid inequality for star bodies, Adv. in Appl. Math., 48 (2012), 432-445. 1

[36] X. Y. Zhu, G. S. Leng, On the $\mathrm{L}_{p}$ intersection body, Appl. Math. Mech., 28 (2007), 1669-1678. 1

[37] B. C. Zhu, N. Li, J. Z. Zhou, Isoperimetric inequalities for $\mathrm{L}_{\mathrm{p}}$-geominimal surface area, Glasgow Math. J., 53 (2011), 717-726.

[38] B. C. Zhu, J. Z. Zhou, W. X. Xu, Lp mixed geominimal surface area, J. Math. Anal. Appl., 422 (2015), 1247-1263. 1 